\title{
- A practical guide and review of EDITOR'S CHOICE colonoscopic surveillance and chromoendoscopy in patients with colitis
}

\author{
Matthew D Rutter
}

University Hospital of North Tees, Stockton-on-Tees, Cleveland, UK

\section{Correspondence to}

Dr Matthew D Rutter, University Hospital of North Tees, Stocktonon-Tees, Cleveland TS19 8PE, UK;

matt.rutter@nth.nhs.uk

Accepted 14 June 2010 Published Online First 28 July 2010

\begin{abstract}
Patients with longstanding extensive colitis have an increased risk of developing colorectal cancer. The British Society of Gastroenterology has recently published revised guidelines for colitis surveillance, taking into account new evidence supporting chromoendoscopy and the endoscopic resection of certain neoplastic lesions. This article reviews some of the evidence behind this paradigm shift and gives a practical guide to chromoendoscopy.
\end{abstract}

\section{Increased colorectal cancer risk and surveillance}

Patients with longstanding extensive colitis, whether Crohn's colitis or ulcerative colitis, have a five to six times higher risk of developing colorectal carcinoma than the general population. ${ }^{12}$ Cancers often occur at a younger age than in the general population, with over a quarter presenting before the age of 40. Colonoscopic surveillance is recommended by most authorities to reduce the associated mortality and recent studies have shown some reduction in cancer incidence, which may in part be due to the effect of surveillance programmes.

Surveillance aims to detect premalignant dysplastic tissue. In non-colitic patients the premalignant lesion, the adenoma, is usually clearly delineated and macroscopically visible. However, in colitis, dysplasia can be subtle, varied and mimicked by inflammatory and postinflammatory changes. Because of this the recommendation has previously been to take multiple random biopsy specimens of flat mucosa. It has been estimated that 33 biopsies are required to have a $90 \%$ chance of finding the highest degree of dysplasia present. $^{3}$
However, recent evidence has demonstrated that random biopsies have a very low yield for dysplasia, ${ }^{4-8}$ and that the policy is costly, time consuming and poorly adhered to.

\section{Chromoendoscopy}

Endoscopic dye-spraying (chromoendoscopy) involves the application of a mucosal stain or pigment, usually by injection down an endoscopic spray-catheter. Two main stains are available for chromoendoscopy: indigo carmine, a contrast dye which simply coats the colonic mucosal surface, highlighting subtle disruption to the normal contours of the colorectum caused by mucosal lesions, and methylene blue, a dye which is absorbed avidly by non-inflamed mucosa, but is poorly taken up by active inflammation and dysplasia, creating a colour contrast. Concern has recently been raised about the safety of methylene blue: one study found in vitro evidence of DNA damage at the concentration used in the colon. ${ }^{9}$ Whether this is of any clinical significance is unclear.

Chromoendoscopy has two main uses in colitis surveillance. First, it improves the detection of subtle colonic lesions, increasing the sensitivity of the endoscopic examination-important with the subtle lesions of colitis dysplasia. Second, once a lesion has been detected, chromoendoscopy can aid differentiation between neoplastic and non-neoplastic lesions by assessing the crypt architecture using the modified pit pattern classification (figure 1 ). ${ }^{10}$

With recent improvements in endoscopic equipment and technique, it has been established that the majority of colitic dysplastic lesions are macroscopically visible and that dysplasia yield is 
significantly enhanced if targeted biopsy specimens are taken after pancolonic dye spraying, ${ }^{4-611}$ reducing the colonoscopist's dependence on random biopsies.

\begin{tabular}{lcll} 
Type & Pit pattern & Definition & $\begin{array}{l}\text { Usual histopatho- } \\
\text { logical findings }\end{array}$ \\
\hline Type I & & round pits & normal \\
& & &
\end{tabular}

Type II

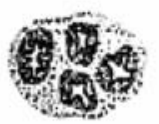

asteroid or papillary pits

hyperplastic

Type IIIs

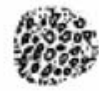

small tubular or roundish pits

intramucosal adenocarcinoma $(28.3 \%)$ adenoma $(73 \%)$ (depressed lesion)

Type IIIL

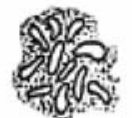

large tubular or roundish pits

adenoma $(86.7 \%)$ (protruded lesion)

Type IV

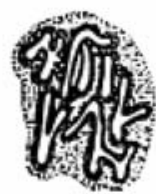

branch-like or gyrus-like pits

a $(59.7 \%)$

(almost tubulovillous adenoma) intramucosal adeno. carcinoma $(37.2 \%)$

Type V

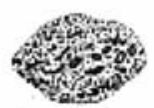

non-structura pits

submucosal adenocarcinoma $(62.5 \%)$

Figure 1 Modified pit pattern classification. Reproduced, with permission, from Kudo S. Endoscopy mucosal resection of flat and depressed types of early colorectal cancer. Endoscopy 1993;25:455-61.
In Kiesslich's study, chromoendoscopy using methylene blue detected three times more dysplasia than surveillance using random biopsies (32/84 vs 10/81; $\mathrm{p}=0.003) .{ }^{6}$ In a study from St Mark's Hospital of backto-back colonoscopies in 100 patients, no dysplasia was detected from 2904 random biopsies, two visible dysplastic lesions were detected macroscopically and a further seven lesions were unmasked by indigo carmine dye-spraying. ${ }^{4}$ The 2010 revised British Society of Gastroenterology guidelines for colitis surveillance recommend chromoendoscopy with targeted biopsy of abnormal areas (figure 2). ${ }^{12}$

Pan-colonic dye-spraying is inexpensive, technically quite easy to perform, quick (adding only a few minutes to the endoscopic procedure compared with random biopsying) and safe.

\section{Other imaging modalities}

Although there was much hope that narrow band imaging (NBI) might prove as effective as chromoendoscopy, a prospective randomised cross-over trial has demonstrated that NBI is no better than standard colonoscopy ('white light endoscopy' (WLE)) and therefore cannot be recommended for colitis surveillance. ${ }^{7}$

The results of a small prospective randomised crossover trial were in favour of another image-enhancement technique, autofluorescence imaging (AFI), when compared with WLE: 10 dysplastic lesions were found in the 25 patients undergoing AFI first, subsequent WLE detecting no further lesions, compared with three dysplastic lesions in the 25 patients undergoing WLE first, subsequent AFI detecting three further lesions $(p=0.036) .{ }^{8}$ No study has yet assessed the technique against pan-colonic dye-spraying.

Confocal endomicroscopy produces real-time in vivo images of the cellular structure of the mucosa.

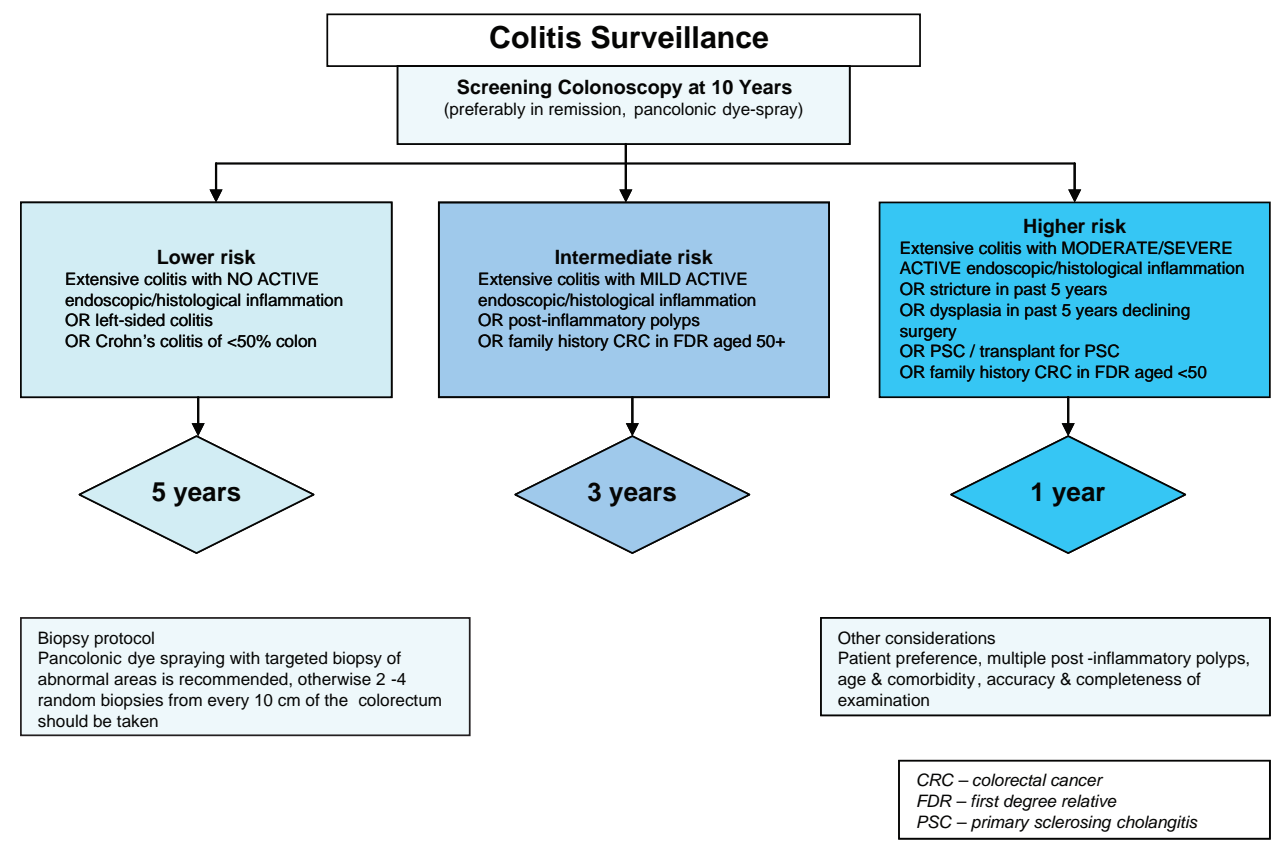

Figure 2 British Society of Gastroenterology colitis surveillance algorithm. ${ }^{12}$ 
However, although confocal endomicroscopy improves lesion characterisation, it is not a technology for lesion detection itself.

\section{Chromoendoscopy technique}

In this section I will describe the techniques I employ for pan-colonic dye-spraying.

Surveillance colonoscopies should ideally be performed during disease remission, to aid the discrimination of inflammatory from neoplastic lesions by both the colonoscopist and the histopathologist. A patient with active symptoms should have their medical treatment optimised first to induce remission. However, procedures should not be delayed unduly if patients fail to respond to treatment as those with ongoing active inflammation have an increased risk of colorectal neoplasia. ${ }^{13}$ As colitis surveillance is the most difficult diagnostic colonoscopic procedure, it should only be performed by colonoscopists skilled in interpreting the colonoscopic appearances.

As the aim is to detect subtle lesions a high-quality bowel preparation regimen, of which many are available, is important. On colonoscope insertion, adherent stool should be washed and residual faecal fluid aspirated as it is more difficult to do this on extubation when the dye-spray catheter occludes the suction channel. Antifoaming agent can be used to dissipate any bubbles which may obscure mucosal views. Any abnormalities seen on insertion should be biopsied or removed as they may not be easily identified on extubation. Single biopsy specimens from the rectum, descending colon, transverse colon and ascending colon should be taken to map the severity/extent of colorectal inflammation.

Once the caecal pole is reached, intravenous buscopan $20 \mathrm{mg}$ should be given to reduce blind-spots by suppressing spasm and haustral fold prominence. Meticulous inspection of the colonic mucosa is performed on withdrawal: there is clear evidence that the more time that is spent inspecting the mucosa, the greater the dysplasia yield. ${ }^{14} 15$

During extubation, adequate distension of the lumen is necessary. This is best achieved by position shifts-in general, the left lateral position provides optimal views of the proximal colon, supine position for the transverse colon and right lateral position for the distal colon. If the lumen remains collapsed despite position shift, further insufflation with air (or preferably carbon dioxide, which is more readily absorbed from the lumen reducing periprocedure and postprocedure patient discomfort) should be used.

Both methylene blue and indigo carmine can be used at a concentration of $0.1 \%$. I use indigo carmine and have found that the most convenient way to prepare the solution is to add six $5 \mathrm{ml}$ vials of indigo carmine $0.4 \%$ to a $100 \mathrm{ml}$ bag of normal saline, creating a concentration of approximately $0.1 \%$, then using $20 \mathrm{ml}$ Luer lock syringes to withdraw the mixed solution-the resultant $130 \mathrm{ml}$ of solution is usually sufficient for one procedure.
Proprietary spray catheters are available (such as the Olympus PW-5V1 disposable washing catheter) which help coat all of the mucosa. The endoscopy assistant needs to apply constant firm pressure on the syringe to generate a mist of dye-too little pressure results in drips of dye from the catheter tip, or unidirectional spray (figure 3).

The colorectum is sprayed segmentally on withdrawal. The length of segment sprayed will vary depending on the tortuosity of the bowel. In general, segments of $5-15 \mathrm{~cm}$ will be sprayed.

Several different dye-spraying techniques can be employed, the aim of each of course being to coat all of the mucosa as effectively as possible. I would estimate that it probably takes around 20-30 cases to master the techniques, but it is easy to judge whether your technique is good or not, depending on how much of the mucosa turns blue!

In the conventional 'spiral' technique, the spraycatheter is protruded about $1-2 \mathrm{~cm}$ beyond the tip of the scope and kept in this position. The colonoscopist then steers the tip of the scope in a spiral fashion on slow withdrawal as the assistant sprays the dye.

In the 'catheter advance' technique, useful for long straight segments of colon such as the transverse or descending colon, the colonoscope is withdrawn to the distal extent of the segment to be sprayed and then the catheter is advanced beyond the tip of the scope to the proximal extent of that segment, before slowly withdrawing it towards the scope while spraying. The colonoscopist should aim preferentially for the mucosa on the wall opposite to fluid pools, allowing gravity to aid circumferential coating of the mucosa with dye.

An alternative catheter-less technique for such segments is to put $5 \mathrm{ml}$ of dye solution in a $50 \mathrm{ml}$ syringe, filling the rest of the syringe with air. The whole syringe is then injected rapidly down the biopsy channel-it can be remarkably effective in coating the mucosa.

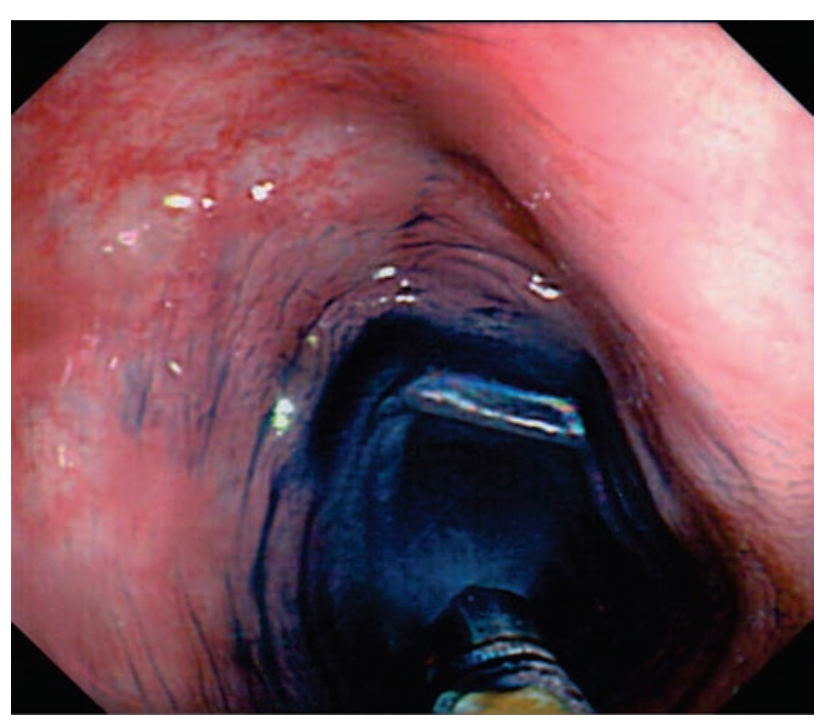

Figure 3 Chromoendoscopy with dye-spray catheter. 
Undoubtedly, areas of mucosa will remain dye-free after initial spraying - these can be easily re-sprayed. Other methods for enhancing mucosal coverage include position shifts and causing a bowel segment to collapse by suctioning the air-the latter technique works well if there is a large pool of dye in that segment.

Once a segment has been sprayed, excess dye should be suctioned, and the colonoscope reinserted to the proximal extent of the segment before meticulous inspection is performed. Careful attention should be paid to ensure that mucosa proximal to colonic folds is not overlooked. It is occasionally necessary to wait a few seconds for indigo carmine to settle into the mucosal pits and grooves; methylene blue takes about $1 \mathrm{~min}$ to be absorbed. Once that segment has been examined, the next segment is sprayed, and so on until the entire colorectum has been inspected.

\section{What you're looking for}

Dysplastic lesions occurring proximal to the extent of colitic inflammation are sporadic adenomas and can be resected endoscopically.

Changes of dysplasia in colitis can be subtle and varied, yet most dysplasia is macroscopically detectable with careful chromoendoscopic inspection. Any break in the normal mucosal contour or colour change from surrounding mucosa before dye-spraying should be scrutinised, paying particular attention to areas of villiform, nodular or friable mucosa. Such areas should be photographed, biopsied and either marked or resected, unless dysplasia can be excluded in vivo by assessment of the crypt architecture (pit pattern). This technique, aided by the use of a magnifying colonoscope and chromoendoscopy, helps the colonoscopist to discriminate neoplastic from non-neoplastic lesions and can reduce the number of biopsies required during a procedure. Neoplastic changes are characterised by an irregular, tubular or villous crypt architecture staining pattern (pit patterns III-V). Non-neoplastic changes are characterised by stellar or regular round pits (pit pattern types I or II) (figure 1).

\section{How to manage dysplasia}

If dysplasia is detected at any surveillance colonoscopy, the histology should be double-reported by an expert gastrointestinal pathologist and the surveillance colonoscopy repeated by an expert colonoscopist using chromoendoscopy (and in this specific situation taking multiple random biopsy specimens as well). The clinician can then determine whether this is clear-cut dysplasia, whether there are multiple lesions and whether they are discrete and endoscopically resectable.

Raised dysplastic lesions arising within an area of current or previous inflammation have been termed dysplasia-associated lesions/masses (DALMs) and early studies showed high cancer incidences in such patients. However, there is no universally agreed definition of a DALM. Recently, the term 'adenomalike mass' (ALM) has been used to describe dysplastic polyps detected within an area of inflammation and judged to be broadly similar in appearance to sporadic adenomas (figure 4). Endoscopic resection of such lesions carries a good prognosis: in one follow-up study of 24 patients, 59\% had developed further dysplastic polyps after a mean follow-up of 82 months, with one patient with primary sclerosing cholangitis developing adenocarcinoma 7.5 years later ${ }^{16}$; in another study, 70 such dysplastic polyps were resected from 48 patients, $48 \%$ developed further dysplastic polyps (no significant difference from a comparator cohort of patients with non-ulcerative colitis following sporadic adenoma polypectomy) and none developed cancer over a mean 4.1 year follow-up ${ }^{17}$; another study of 40 patients reported one case of adenocarcinoma after 4.2 years mean follow-up, not significantly different from the frequency of cancer within the surveillance population as a whole $(\mathrm{p}=1.0$, Fisher's exact test $) .{ }^{18}$ As there is no clear-cut histological discriminator between socalled 'DALMs' and 'ALMs', management depends on whether the lesion can be endoscopically resected: if it can be resected in its entirety (with chromoendoscopic demarcation of the edge of the lesion biopsies taken from the surrounding mucosa to ensure there is no residual dysplasia), it is often not necessary to recommend colectomy. Otherwise urgent reassessment of resectability by an experienced colonoscopist or urgent surgery is required-particularly as unresectable lesions often harbour cancer despite negative superficial biopsies.

Where endoscopically invisible high-grade dysplasia is detected, colectomy is advised owing to high rates of synchronous cancer or rapid progression to cancer in $32-42 \%$ of patients. ${ }^{19}$ Management of endoscopically invisible low-grade dysplasia is

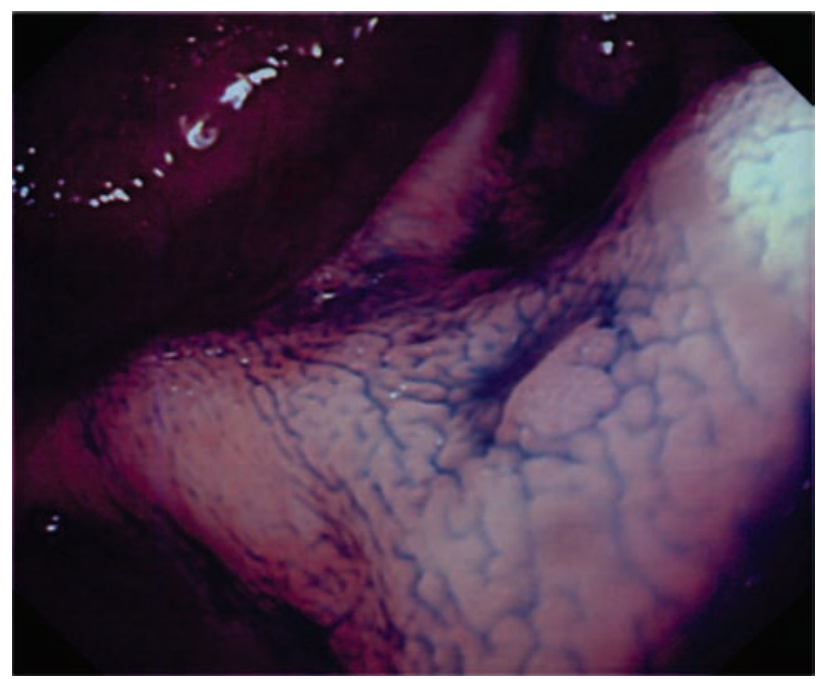

Figure $4 \quad 3 \mathrm{~mm}$ well-circumscribed low-grade dysplastic lesion. 
fraught with controversy as reported rates of progression to high-grade dysplasia or cancer vary from as low as $3 \%$ to over $50 \% .{ }^{20-23}$ The options of either colectomy or intensified surveillance needs to be discussed frankly with the patient, taking into account other risk factors.

\section{Postinflammatory polyps}

Patients with multiple postinflammatory polyps present a clinical dilemma as they have a twofold risk of colorectal cancer, ${ }^{24}$ yet the colonoscopic detection of subtle neoplasia in the presence of multiple postinflammatory polyps can be extremely difficult. The endoscopist should look for, and biopsy or remove, any atypical polypoid lesion-for example, one which does not have a smooth mucosal surface. The clinician should have a full and frank discussion with the patient about other potential management strategies such as prophylactic surgery.

\section{Conclusion}

Colonoscopic surveillance for neoplasia in colitis has moved into a new era. With better endoscopic detection techniques, better understanding of the natural history of dysplasia and opportunities for the endoscopic resection of certain lesions, the hope is that we can further reduce cancer incidence while also reducing the need for colectomy.

Competing interests MDR is co-author of BSG colitis surveillance guidelines.

Provenance and peer review Commissioned; externally peer reviewed.

\section{References}

1. Ekbom A, Helmick C, Zack M, et al. Ulcerative colitis and colorectal cancer. A population-based study. N Engl J Med 1990;323:1228-33.

2. Ekbom A, Helmick C, Zack M, et al. Increased risk of largebowel cancer in Crohn's disease with colonic involvement. Lancet 1990;336:357-9.

3. Rubin CE, Haggitt RC, Burmer GC, et al. DNA aneuploidy in colonic biopsies predicts future development of dysplasia in ulcerative colitis. Gastroenterology 1992;103:1611-20.

4. Rutter MD, Saunders BP, Schofield G, et al. Pancolonic indigo carmine dye spraying for the detection of dysplasia in ulcerative colitis. Gut 2004;53:256-60.

5. Marion JF, Waye JD, Present DH, et al. Chromoendoscopytargeted biopsies are superior to standard colonoscopic surveillance for detecting dysplasia in inflammatory bowel disease patients: a prospective endoscopic trial. Am J Gastroenterol 2008;103:2342-9.

6. Kiesslich R, Fritsch J, Holtmann M, et al. Methylene blue-aided chromoendoscopy for the detection of intraepithelial neoplasia and colon cancer in ulcerative colitis. Gastroenterology 2003;124:880-8.
7. Dekker E, van den Broek FJ, Reitsma JB, et al. Narrow-band imaging compared with conventional colonoscopy for the detection of dysplasia in patients with longstanding ulcerative colitis. Endoscopy 2007;39:216-21.

8. van den Broek FJ, Fockens P, van Eeden S, et al. Endoscopic tri-modal imaging for surveillance in ulcerative colitis: randomised comparison of high-resolution endoscopy and autofluorescence imaging for neoplasia detection; and evaluation of narrow-band imaging for classification of lesions. Gut 2008;57:1083-9.

9. Davies J, Burke D, Olliver JR, et al. Methylene blue but not indigo carmine causes DNA damage to colonocytes in vitro and in vivo at concentrations used in clinical chromoendoscopy. Gut 2007;56:155-6.

10. Kudo S, Tamura S, Nakajima T, et al. Diagnosis of colorectal tumorous lesions by magnifying endoscopy. Gastrointest Endosc 1996;44:8-14.

11. Matsumoto T, Nakamura S, Jo Y, et al. Chromoscopy might improve diagnostic accuracy in cancer surveillance for ulcerative colitis. Am J Gastroenterol 2003;98:1827-33.

12. Cairns S, Scholefield JH. Guidelines for colorectal cancer screening in high risk groups. Gut 2002;51(Suppl 5):V1-2.

13. Rutter M, Saunders B, Wilkinson K, et al. Severity of inflammation is a risk factor for colorectal neoplasia in ulcerative colitis. Gastroenterology 2004;126:451-9.

14. Barclay RL, Vicari JJ, Doughty AS, et al. Colonoscopic withdrawal times and adenoma detection during screening colonoscopy. N Engl J Med 2006;355:2533-41.

15. Toruner M, Harewood GC, Loftus EV Jr, et al. Endoscopic factors in the diagnosis of colorectal dysplasia in chronic inflammatory bowel disease. Inflamm Bowel Dis 2005;11:428-34.

16. Odze RD, Farraye FA, Hecht JL, et al. Long-term follow-up after polypectomy treatment for adenoma-like dysplastic lesions in ulcerative colitis. Clin Gastroenterol Hepatol 2004;2:534-41.

17. Rubin PH, Friedman S, Harpaz N, et al. Colonoscopic polypectomy in chronic colitis: conservative management after endoscopic resection of dysplastic polyps. Gastroenterology 1999;117:1295-300.

18. Rutter MD, Saunders BP, Wilkinson KH, et al. Most dysplasia in ulcerative colitis is visible at colonoscopy. Gastrointest Endosc 2004;60:334-9.

19. Bernstein CN, Shanahan F, Weinstein WM. Are we telling patients the truth about surveillance colonoscopy in ulcerative colitis? Lancet 1994;343:71-4.

20. Befrits R, Ljung T, Jaramillo E, et al. Low-grade dysplasia in extensive, long-standing inflammatory bowel disease: a followup study. Dis Colon Rectum 2002;45:615-20.

21. Lim CH, Dixon MF, Vail A, et al. Ten year follow up of ulcerative colitis patients with and without low grade dysplasia. Gut 2003;52:1127-32.

22. Ullman T, Croog V, Harpaz N, et al. Progression of flat low-grade dysplasia to advanced neoplasia in patients with ulcerative colitis. Gastroenterology 2003;125:1311-19.

23. Connell WR, Lennard-Jones JE, Williams CB, et al. Factors affecting the outcome of endoscopic surveillance for cancer in ulcerative colitis. Gastroenterology 1994;107:934-44.

24. Rutter MD, Saunders BP, Wilkinson KH, et al. Cancer surveillance in longstanding ulcerative colitis: endoscopic appearances help predict cancer risk. Gut 2004;53:1813-16. 